\title{
La ergonomía y los métodos de evaluación de carga postura
}

\section{Ergonomics and postural load assessment methods}

1 Yelena Tamara Chávez Cujilán.

Universidad de Guayaquil, Guayaquil, Ecuador yelena.chavezc@ug.edu.ec

2 Betsy Mabel Moran Olvera Universidad de Guayaquil, Guayaquil Ecuador betsy.olveram@ug.edu.ec
iD https://orcid.org/0000-0002-3989-9936

https://orcid.org/0000-0003-4644-8209

Artículo de Investigación Científica y Tecnológica

Enviado: 11/12/2021

Revisado: $27 / 12 / 2021$

Aceptado: 28/01/2022

Publicado:05/02/2022

DOI: https://doi.org/10.33262/ap.v4i1.1.159

\section{Chávez Cujilán, Y. T., \& Moran Olvera, B. M. (2022). La ergonomía y los métodos de} evaluación de carga postura . AlfaPublicaciones, 4(1.1), 279-292. https://doi.org/10.33262/ap.v4i1.1.159

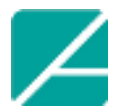

Ciencia

Digital

ALFA PUBLICACIONES, es una Revista Multidisciplinar, Trimestral, que se publicará en soporte electrónico tiene como misión contribuir a la formación de profesionales competentes con visión humanística y crítica que sean capaces de exponer sus resultados investigativos y científicos en la misma medida que se promueva mediante su intervención cambios positivos en la sociedad. https://alfapublicaciones.com

La revista es editada por la Editorial Ciencia Digital (Editorial de prestigio registrada en la Cámara Ecuatoriana de Libro con No de Afiliación 663) www.celibro.org.ec

Esta revista está protegida bajo una licencia Creative Commons Attribution Non Commercial No Derivatives

4.0 International. Copia de la licencia: http://creativecommons.org/licenses/by-nc-nd/4.0/ 


\section{Palabras \\ claves:}

ergonomía, OWAS, REBA, RULA, EPR

\section{Keywords:} ergonomics, OWAS, REBA, RULA, EPR.

\section{Resumen}

La ergonomía es una de las ciencias o disciplinas que apoyan a la gestión de la higiene y seguridad de una empresa, esta permite evaluar los factores de riesgos a nivel de puestos de trabajo y la interacción entre el trabajador y las herramientas y entorno laboral, con la finalidad de establecer los posibles riesgos que afecten a la salud de los trabajadores y puedan generar trastornos en la salud de los mismos. El presente estudio se realizó basado en la ergonomía, sus definiciones y características, así como definir los métodos enfocados en la evaluación de carga postural como lo son el método RULA, REBA. OWAS y Evaluación Postural Rápida (EPR), los cuales aportan al investigador herramientas para evaluar las incidencias y relación entre las herramientas de trabajo y las posturas de los trabajadores, con la finalidad de conocer su metodología de aplicación, por medio de una investigación bibliográfica.

\section{Abstract}

Ergonomics is one of the sciences or disciplines that support the hygiene and safety management of a company, it allows evaluating risk factors at the level of jobs and the interaction between the worker and the tools and work environment, with The purpose of establishing the possible risks that affect the health of workers and may generate health problems for them. The present study was carried out based on ergonomics, its definitions and characteristics, as well as defining the methods focused on the evaluation of postural load such as the RULA, REBA method. OWAS and Rapid Postural Evaluation (EPR), which provide the researcher with tools to evaluate the incidents and relationship between the work tools and the positions of the workers, in order to know their application methodology, through a bibliographic investigation.

\section{Introducción}

Las empresas a nivel global han experimentado diversos cambios a lo largo de los años, se ha visto como las necesidades del ser humano han cambiado en función de las expectativas de los mercados internacionales, las grandes empresas operan con una maquinaria publicitaria que impulsa al consumidor a necesitar cosas que quizás no tienen tanta importancia para su vida, sin embargo esto ha provocado que los mercados a nivel 
mundial estén al tope de las demandas y por ende la producción de bienes y servicios se torna cada vez más caótica y demandante. Las crecientes demandas de productos y servicios precisa de empresas altamente competitivas y con una capacidad de producción y productividad alta, por lo cual los ritmos laborales para cubrir dichos requerimientos son cada vez más pesados y demandantes, si bien es cierto que gran parte de las grandes transnacionales han automatizado una buena parte de sus procesos también lo es que el capital humano aún sigue siendo el principal motor que mueve a las empresas y organizaciones en todo el mundo, por esta razón es de suma importancia que los trabajadores puedan contar con una buena salud y con todas las garantías que les permita realizar sus labores con toda la seguridad y el confort necesario, sin que esto vaya en menoscabo de su salud ni de sus capacidades físicas ni emocionales.

De acuerdo a lo anterior, es importante mencionar que la Higiene y Seguridad Laboral se encarga de la evaluación y el control de todos aquellos factores del lugar de trabajo que pueden causar enfermedades y deteriorar la salud, así mismo, de adelantar procesos para la protección de los trabajadores, el monitoreo médico y la incorporación de controles técnicos y de capacitación vinculada a la previsión y seguimiento de riesgos, acción de la cual se ocupa la seguridad industrial (Ortega et al., 2018). Esta disciplina es una de las fundamentales a la hora de realizar un monitoreo sobre las condiciones laborales de una determinada empresa.

En este caso, se realiza una investigación de tipo bibliográfica la cual busca plasmar una de las disciplinas que complementan a la higiene y seguridad laboral, como lo es la ergonomía haciendo énfasis en los diferentes métodos para la evaluación de los puestos de trabajo, esto dará al lector una visión más completa de los métodos más comunes a usar en la carga postural en la búsqueda del confort en los entornos laborales, las características principales de los métodos, como y cuando deben ser usados, así como las generalidades de cada caso.

\section{Metodología}

La presente investigación se presenta bajo la metodología de revisión bibliográfica, la cual tiene como propósito realizar consultas de diferentes autores con la finalidad de generar una serie de conclusiones y discusión de los resultados, la revisión de la literatura implica detectar, consultar y obtener la bibliografía (referencias) y otros materiales que sean útiles para los propósitos del estudio, de donde se tiene que extraer y recopilar la información relevante y necesaria para enmarcar nuestro problema de investigación (Vásquez, 2020).

Por otro lado, es de tipo documental, ya que el investigador realiza una búsqueda de información de segunda mano, cuando busca y elige aquella información que ya está documentada: registrada, recopilada y clasificada; información que puede estar en forma 
de escritura, voz, imagen, sonido, símbolos gráficos, tablas o cuadros estadísticos, mapa, dibujo, escultura, etc. (Vásquez, 2020).

La presente investigación se realizó bajo la revisión de diferentes artículos, libros, trabajos de grado, proyectos, revistas científicas y fuentes verificables que garantizan la fiabilidad de los conceptos y análisis que se presentan.

\section{Resultados y Discusión}

Desde los primeros hombres en la tierra, los instrumentos de caza y recolección se elaboraron con la finalidad de ayudar a los hombres a hacer sus tareas diarias más fáciles, la evidencia arqueológica sugiere que los primeros habitantes de la tierra seleccionaban las piedras que mejor se adaptaban para realizar trabajos que les proporcionaran comida y un lugar donde vivir, ellos buscaban que la vivienda, el vestido y su entorno cumpliera con algo de comodidad y cumpliera con las necesidades que en ese momento de la historia aquejaban a los habitantes.

Esta situación se mantuvo por largos años pero en constante evolución, a medida que pasaban los años, los hombres adquirían experiencia en sus labores llegando a perfeccionar sus técnicas de caza y elaboración de herramientas para sus tareas, todo esto cumpliendo básicamente dos premisas, la primera que resultaran instrumentos e indumentaria útil para la ardua tarea de buscar comida y cubrir sus cuerpos para protegerlos de las condiciones climáticas y de animales, y por otro lado que resultaran cómodos para su mejor uso y mantener el cuerpo abrigado y protegido, esto no es más que un instinto para la supervivencia que el ser humano posee, pero que va enfocado a buscar la mejora de sus situaciones buscando mantener cierta comodidad para él y los suyos.

Esta búsqueda fue cada día avanzando a medida que surgían civilizaciones en las diferentes regiones del mundo, es de notar las egipcias, romana y griega, en la que los avances en distintas áreas fue considerable y dio impulso a muchos de los grandes descubrimientos que hoy en día aún persisten, estas civilizaciones edificaron grandes monumentos usando mano de obra esclava, irónicamente para la búsqueda de su comodidad sin tomar en cuenta las deplorables condiciones de insalubridad y desconformidad en los trabajadores, para Hernández (2014 citado por Delado et al., 2017), la ergonomía nació con la actividad humana, pensada y dirigida a metas. Hace más de 2500 años antes de enunciarse el concepto de ergonomía, parece que sus principios básicos se utilizaron en la Grecia antigua, de los que quedaron como póstumo homenaje sus utensilios, la forma en que concibieron sus teatros y las ventajas que brindaban a sus venerados ancianos. 
Luego, más adelante en la era de la máquina de vapor, la producción en masa alcanzo niveles muy altos debido a la alta demanda de productos, aunado a esto la carrera militar y armamentista marco una importante referencia en la promoción de la ergonomía en el mundo, ya que las armas, aviones e indumentaria comenzaron a ser diseñados con miras a aportar comodidad a los soldados al usarlos, debido a las largas y extenuantes jornadas de los soldados en las guerras, dando los primero pasos para la consolidación de la disciplina ergonomía.

La idea fue introducida en 1949 por el psicólogo británico K.F.H. Murrell, cuando un grupo de científicos se reunió en Inglaterra para formar la Sociedad de Investigaciones ergonómicas. La idea fue cobijar bajo el mismo alero a ingenieros, fisiólogos, anatomistas, psicólogos, higienistas industriales, arquitectos, profesionales del área de la salud y en general personas interesadas en el comportamiento humano en el trabajo (Delado et al., 2017).

La ergonomía es una disciplina científico-técnica y de diseño que estudia la relación entre el entorno de trabajo (lugar de trabajo), y quienes realizan el trabajo (los trabajadores). Dentro del mundo de la prevención es una técnica preventiva que intenta adaptar las condiciones y organización del trabajo al individuo. Su finalidad es el estudio de las personas en su trabajo y tiene como propósito ultimo conseguir el mayor grado de adaptación o ajuste, entre ambos. Su objetivo es hacer el trabajo lo más eficaz y cómodo posible (Tongombol \& Cartolin, 2019).

La palabra ergonomía - "la ciencia del trabajo" se deriva del griego "ergon" (trabajo) y nomos (leyes). La ergonomía (o factores humanos) es la disciplina científica que se ocupa de la comprensión de las interacciones entre humanos y otros elementos de un sistema, y la profesión que aplica teoría, principios, datos y métodos para diseñar con el fin de optimizar el bienestar humano y general (IEA, 2020).

Para la Asociación Española de Ergonomía (AEE, 2020), la ergonomía es el conjunto de conocimientos de carácter multidisciplinar aplicados para la adecuación de los productos, sistemas y entornos artificiales a las necesidades, limitaciones y características de sus usuarios, optimizando la eficacia, seguridad y bienestar. En este sentido Cruz \& Garnica (2010) menciona que:

El propósito de la ergonomía es reconocer y estudiar los parámetros que serán utilizados de manera real y práctica, para ser aplicados en el planteamiento de la solución de un objeto requerido por un grupo, además de esto la ergonomía es científica e interdisciplinaria, los preceptos están concebidos con base en la racionalidad y objetividad, y se vale de otras disciplinas para complementar los temas. 
La ergonomía se compone de múltiples disciplinas que se complementan para realizar la correcta reingeniería de los procesos y herramientas del trabajo y del entorno que lo rodea, con la finalidad de adaptarlos de una mejor manera a los seres humanos y así mitigar el impacto que puedan tener estos elementos a la salud de los trabajadores, como toda ciencia tiene un objetivo claro y es el de adaptar el trabajo a las capacidades y posibilidades del ser humano, de igual manera el diseño juega un papel fundamental ya que todos los elementos de trabajo ergonómicos se diseñan teniendo en cuenta quiénes van a utilizarlos. Lo mismo debe ocurrir con la organización de la empresa: es necesario diseñarla en función de las características y las necesidades de las personas que las integran (AEE, 2020).

De acuerdo a lo anteriormente expuesto, se puede mencionar otro termino conocido como disergonómia, la cual es una desviación de lo aceptable como ergonómico o confortable para la persona en su labor, es decir, implica aquellos cuadros de riesgos inadecuados del sistema hombre - máquina, desde el punto de vista de diseño, construcción, operación, ubicación de maquinaria, conocimientos, habilidades, condiciones, así como de las características de los operarios, y de las interrelaciones tanto con el entorno como con el medio ambiente de trabajo, tales como: Monotonía, fatiga, malas posturas, movimientos repetitivos y sobrecarga física (Neusa et al., 2019). Estas desviaciones son producto de procesos y herramientas mal diseñadas, las cuales solo fueron concebidas para realizar la labor sin considerar el factor humano y sus diferentes características.

Una de las características de la ergonomía es que se compone de múltiples disciplinas, y de igual manera se vale de diferentes métodos para la evaluación y diagnóstico de situaciones que puedan representar riesgos a la salud de los trabajadores, para el caso de esta investigación se enfocara en la higiene postural y la interacción entre el ser humano y estas, seguidamente se evidencias estos métodos y sus principales características y modo de aplicación.

Los métodos que se enfocan a la determinación de factores de riesgos presentes en los puestos de trabajo y que afectan o pueden afectar a la salud de los trabajadores son los siguientes:

- Método OWAS

- Método REBA

- Método RULA

- Método de Evaluación de Posturas Rápidas

Método Ovako Working Posture Analysis System (OWAS): Para la secretaria de Salud Laboral de Madrid (2016) citado por Escalante et al., (2018) indica que el método OWAS, es un método sencillo y útil destinado al análisis ergonómico de la carga postural; en función de sus fundamentos teóricos se han desarrollado propuestas informáticas lo que 
conlleva a ser el método para medir la carga postural preferido. En su forma tradicional, el método OWAS permite adquirir las observaciones de las diversas posturas, codificarlas, atribuir la clase de riesgo relativo y calcular el porcentaje con el que cada clase se repite en la realización de una determinada actividad o en las diferentes fases en las que ésta ha sido eventualmente subdividida.

A continuación, se describen los pasos para la aplicación del método OWAS descritos por (Diego, 2015):

- Determinar si la tarea debe ser dividida en varias fases (evaluación simple o multifase). Si las actividades desarrolladas por el trabajador son muy diferentes en diversos momentos de su trabajo se llevará a cabo una evaluación multifase.

- Establecer el tiempo total de observación de la tarea dependiendo del número y frecuencia de las posturas adoptadas. Habitualmente oscilará entre 20 y 40 minutos.

- Determinar la frecuencia de observación o muestreo. Indicar cada cuánto tiempo se registrará la postura del trabajador. Habitualmente oscilará entre 30 y 60 segundos.

- Observación y registro de posturas. Observación de la tarea durante el periodo de observación definido y registro las posturas a la frecuencia de muestreo establecida. Pueden tomarse fotografías o vídeos desde los puntos de vista adecuados para realizar las observaciones. Para cada postura se anotará la posición de la espalda, los brazos y las piernas, así como la carga manipulada y la fase a la que pertenece si la evaluación es multifase.

- Codificación de las posturas observadas. A cada postura observada se le asignará un Código de postura que dependerá de la posición de cada miembro y la carga. Se emplearán para ello las tablas correspondientes a cada miembro,

- Calculo de la Categoría de riesgo de cada postura. A partir de su Categoría de riesgo se identificarán aquellas posturas críticas o de mayor nivel de riesgo para el trabajador.

- Cálculo del porcentaje de repeticiones o frecuencia relativa de cada posición de cada miembro. Se calculará el porcentaje de cada posición de cada miembro (espalda, brazos y piernas) respecto al total de posturas adoptadas.

- Cálculo de la Categoría de riesgo para cada miembro en función de la frecuencia relativa. Se conocerá así qué miembros soportan un mayor riesgo y la necesidad de rediseño de la tarea.

- Determinar, en función de los resultados obtenidos, las acciones correctivas y de rediseño necesarias

- En caso de haber introducido cambios, evaluar de nuevo la tarea con el método Owas para comprobar la efectividad de la mejora. 
Método REBA (Rapid Entire Body Assessment): el método REBA evalúa posturas individuales y no conjuntos o secuencias de posturas, por ello, es necesario seleccionar aquellas posturas que serán evaluadas de entre las que adopta el trabajador en el puesto. Se seleccionarán aquellas que, a priori, supongan una mayor carga postural bien por su duración, bien por su frecuencia o porque presentan mayor desviación respecto a la posición neutra. Para ello, el primer paso consiste en la observación de las tareas que desempeña el trabajador. Se observarán varios ciclos de trabajo y se determinarán las posturas que se evaluarán. Si el ciclo es muy largo o no existen ciclos, se pueden realizar evaluaciones a intervalos regulares. En este caso se considerará, además, el tiempo que pasa el trabajador en cada postura (Aguaysa, 2019).

De igual manera se mencionan algunas características del Método REBA y se muestran en la tabla 1:

\section{Tabla 1}

\section{Características del método REBA}

\begin{tabular}{l}
\hline \multicolumn{1}{c}{ Características } \\
\hline Es un método especialmente sensible a los riesgos de tipo músculo-esquelético. \\
Divide el cuerpo en segmentos para ser codificados individualmente, y considera tanto los miembros \\
superiores, como el tronco, el cuello y las piernas. \\
Analiza la repercusión sobre la carga postural del manejo de cargas realizado con las manos o con otras \\
partes del cuerpo. \\
Considera el tipo de agarre de la carga manejada. \\
Permite la valoración de la actividad muscular causada por posturas estáticas, dinámicas, o debidas \\
a cambios bruscos o inesperados en la postura. \\
El resultado determina el nivel de riesgo de padecer lesiones estableciendo el nivel de acción requerido \\
y la urgencia de la intervención.
\end{tabular}

Fuente: Aguaysa (2019)

La aplicación de este método se describe a continuación según Diego (2015):

- Determinar los ciclos de trabajo y observar al trabajador durante varios de estos ciclos. Si el ciclo es muy largo o no existen ciclos, se pueden realizar evaluaciones a intervalos regulares.

- Seleccionar las posturas que se evaluarán. Se seleccionarán aquellas que, a priori, supongan una mayor carga postural bien por su duración, bien por su frecuencia o porque presentan mayor desviación respecto a la posición neutra.

- Determinar si se evaluará el lado izquierdo del cuerpo o el derecho. En caso de duda se analizarán los dos lados.

- Tomar los datos angulares requeridos. Pueden tomarse fotografías desde los puntos de vista adecuados para realizar las mediciones.

- Determinar las puntuaciones para cada parte del cuerpo. Empleando la tabla correspondiente a cada miembro. 
- Obtener las puntuaciones parciales y finales del método para determinar la existencia de riesgos y establecer el Nivel de Actuación.

- Si se requieren, determinar qué tipo de medidas deben adoptarse. Revisar las puntuaciones de las diferentes partes del cuerpo para determinar dónde es necesario aplicar correcciones.

- Rediseñar el puesto o introducir cambios para mejorar la postura si es necesario

- En caso de haber introducido cambios, evaluar de nuevo la postura con el método REBA para comprobar la efectividad de la mejora.

A continuación, se muestra en la Figura 1 y Figura 2, la medición de ángulos REBA y el grupo de miembros en REBA respectivamente.

\section{Figura 1}

Medición de ángulos REBA

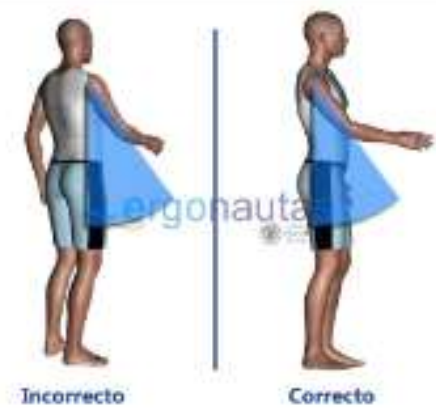

Fivgura ?

Modrión do angyiks en REAA

Fuente: Diego (2015)

\section{Figura 2}

Grupo de miembros en REBA

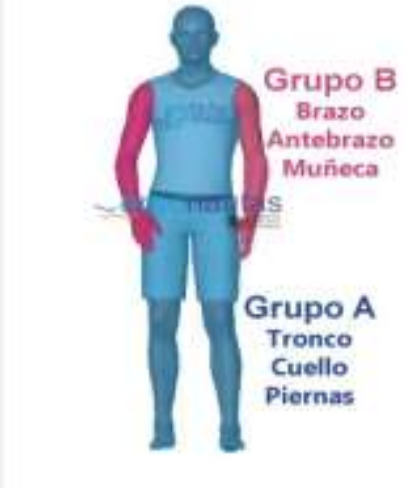

nequa z

grupos de miembren en PSFM

Fuente: Diego J (2015) 
Método RULA (Rapid UpperLimbAssessment): el método RULA es una técnica de evaluación ergonómica. Estudia las posturas individuales y sus factores de riesgo ocupacionales que han sido asociados con desordenes musculoesqueléticos. El uso de RULA da como resultado un factor de riesgo entre 1 y 7 , donde la puntuación o factor más alto significa un gran riesgo. Como quiera que sea, una baja puntuación o factor de riesgo no garantiza que el sitio de trabajo esté libre de riesgos ocupacionales (Naranjo et al., 2020).

Para Asensio et al. (2012, citado por Naranjo et al., 2020), el método RULA permite evaluar lo siguiente:

- El riesgo al que se expone un trabajador al adoptar una determinada postura.

- La exposición al riesgo derivada del levantamiento de cargas en posturas inadecuadas.

- El riesgo causado por la combinación de diferentes posturas críticas.

- La exposición al riesgo de un trabajador que realiza tareas repetitivas con los miembros superiores.

Los pasos para la aplicación de este método son proporcionados por Diego-Mas (2015) y se describen a continuación:

- Determinar los ciclos de trabajo y observar al trabajador durante varios de estos ciclos. Si el ciclo es muy largo o no existen ciclos, se pueden realizar evaluaciones a intervalos regulares.

- Seleccionar las posturas que se evaluarán. Se seleccionarán aquellas que, a priori, supongan una mayor carga postural bien por su duración, bien por su frecuencia o porque presentan mayor desviación respecto a la posición neutra.

- Determinar si se evaluará el lado izquierdo del cuerpo o el derecho. En caso de duda se analizarán los dos lados.

- Tomar los datos angulares requeridos. Pueden tomarse fotografías desde los puntos de vista adecuados para realizar las mediciones.

- Determinar las puntuaciones para cada parte del cuerpo. Empleando la tabla correspondiente a cada miembro.

- Obtener las puntuaciones parciales y finales del método para determinar la existencia de riesgos y establecer el Nivel de Actuación

- Si se requieren, determinar qué tipo de medidas deben adoptarse. Revisar las puntuaciones de las diferentes partes del cuerpo para determinar dónde es necesario aplicar correcciones.

- Rediseñar el puesto o introducir cambios para mejorar la postura si es necesario

- En caso de haber introducido cambios, evaluar de nuevo la postura con el método RULA para comprobar la efectividad de la mejora. 
Método de Evaluación Rápida EPR: EPR no es en sí un método que permita conocer los factores de riesgo asociados a la carga postural, si no, más bien, una herramienta que permite realizar una primera y somera valoración de las posturas adoptadas por el trabajador a lo largo de la jornada. 1 método mide la carga estática considerando el tipo de posturas que adopta el trabajador y el tiempo que las mantiene, proporcionando un valor numérico proporcional al nivel de carga. A partir del valor de la carga estática el método propone un Nivel de Actuación entre 1 y 5.

EPR no evalúa posturas concretas si no que realiza una valoración global de las diferentes posturas adoptadas y del tiempo que son mantenidas. El método considera que el trabajador puede adoptar 14 posibles posturas genéricas, las cuales se muestran en la Figura 3:

\section{Figura 3}

Tabla de Posturas

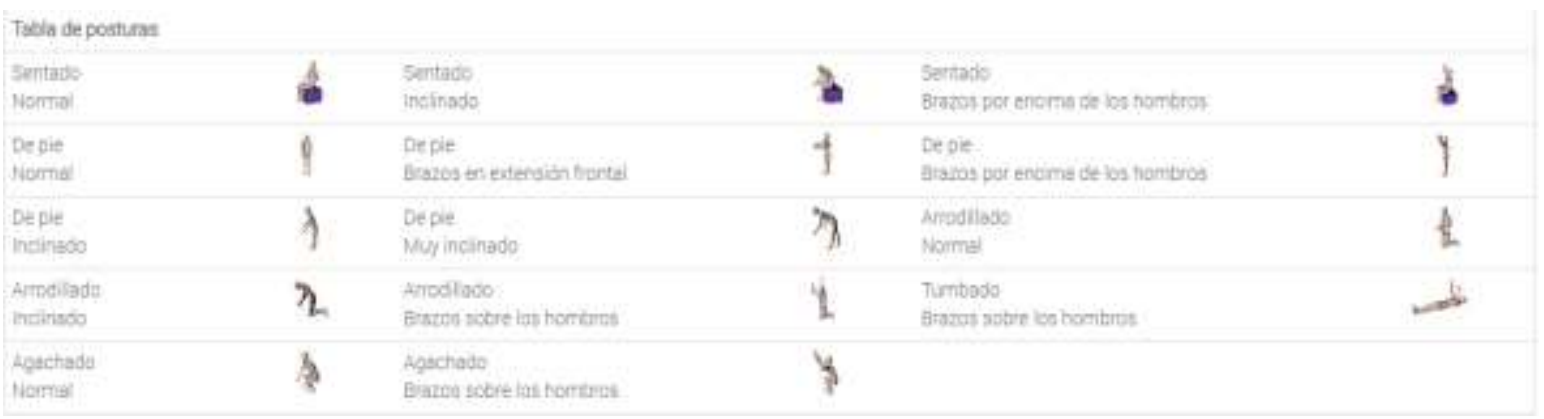

Fuente: Diego (2015)

Los métodos antes descritos son el resultado de los aportes de médicos, enfermeros, psicólogos, ingenieros, entre otros profesionales que dieron sus aportes para crear una metodología que permitiera a los profesionales de la higiene y seguridad laboral realizar la tan importante tarea de evaluar los efectos de los malos diseños de herramientas de trabajo y los efectos en la salud de los trabajadores, esta labor incide en los futuros diseños y en el adiestramiento al personal en mantener una correcta higiene postural, y así de esta manera poder evitar posibles lesiones, que en muchos de los casos desencadena en enfermedades crónicas e incluso la incapacidad total del trabajador.

\section{Conclusiones}

- La ergonomía ha demostrado no solo ser una ciencia que estudia las posturas o malas posturas de las personas en su entorno laboral, ha demostrado que es una disciplina que se complementa de múltiples métodos como la medicina y la psicología para realizar los aportes a las nuevas tendencias en diseños empresariales pensados para el confort de los trabajadores y hacer que sus 
procesos sean amigables con la salud de los trabajadores, y de esta manera incidir positivamente en la productividad empresarial.

- Los métodos para la evaluación, desde el punto de vista ergonómico son variados y resultantes de la gran cantidad de reportes de trastornos musculo esqueléticos que durante décadas han sufrido los empleados de diferentes empresas, industrias o cualquier centro de trabajo.

- Para efectos de este estudio se revisó la diferente bibliografía existente sobre los cuatro métodos dedicados a la evaluación postural, con la finalidad de conocer acerca de las definiciones y las metodologías a emplear.

\section{Referencias Bibliográficas}

Aguaysa, P. (2019). Posturas de trabajo y su relación con la sintomatología de dolor lumbar en docentes de enseñanza Primaria General - Nivel Inicial. Ambato: Proyecto de Trabajo de Investigación, previo a la obtención del Grado Académico de Magister en Seguridad e Higiene Industrial y Ambiental.

Cruz, J., \& Garnica, G. (2010). Ergonomía Aplicada. Bogotá: Eco Ediciones.

Delado, M., Cuichan, D., \& Sancan, M. (2017). Algunas especificidades acerca de la Ergonomía y los factores de riesgo en salud ocupacional. Polo del Conocimiento, 1220-1229.

Diego, J. (2015). Evaluación Postural mediante el método REBA. Recuperado el 07 de enero de 2022, de Ergonautas, Universidad Politécnica de Valencia: https://www.ergonautas.upv.es/metodos/reba/reba-ayuda.php

Diego, J. (2015). Evauación postural mediante el Método OWAS.

Diego-Mas, J. (2015). Evaluacion Postural Mediante el Método RULA.

Escalante, M., Nuñez, M., \& Izquierdo, H. (2018). Evaluación ergonómica en la producción. Caso de estudio: Sector Aluminio, Estado Bolívar. Venezuela. Ingeniería Industrial. Actualidad y Nuevas Tendencias, 73-90.

IEA. (2020). Asociacion Internacional de Ergonomía.

Naranjo, A., Ramírez, E., López, M., \& Rodríguez, I. (2020). Manual de prácticas de laboratorio de ergonomía. Ciudad Obregón: Instituto Tecnológico de Sonora.

Neusa, G., Alvear, R., Cabezas, E., \& Jiménez, J. (2019). Riesgos disergonómicos: Biometría postural de los trabajadores de plantas industriales en Ecuador. Revista de Ciencias Sociales 25(1), 415-428. 
Ortega, J., Rodríguez, J., \& Hernández, H. (2018). Importancia de la seguridad de los trabajadores en el cumplimiento de procesos, procedimientos y funciones. Revista Academia \& Derecho, 155-176.

Tongombol, D., \& Cartolin, F. (2019). Evaluación de riesgos ergonómicos aplicando los métodos OWAS Y REBA en los puestos de trabajo de la empresa MAXLIM S.R.L -. Lima: Tesis para obtener el titulo de ingeniero ambiental de la Universidad Peruana Unión.

Vásquez, W. (2020). Metodología de la Investigación. Manual del Estudiante. Universidad San Martin de Porres. 
El artículo que se publica es de exclusiva responsabilidad de los autores y no necesariamente reflejan el pensamiento de la Revista Alfa Publicaciones.

\section{Ciencia}

El artículo queda en propiedad de la revista y, por tanto, su publicación parcial y/o total en otro medio tiene que ser autorizado por el director de la Revista Alfa Publicaciones.
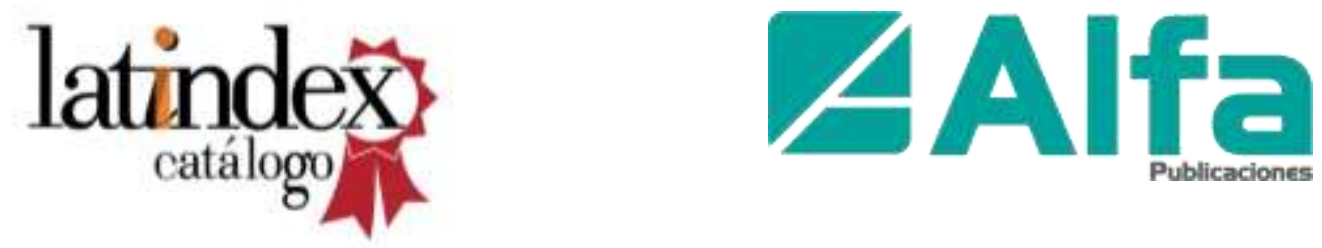

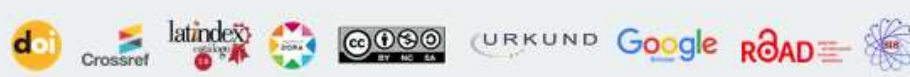
DLatinREV

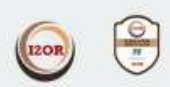

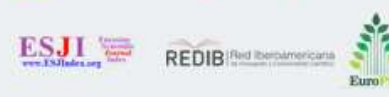
$\mathbf{W}_{\text {wizdom.ai }}$<smiles></smiles>

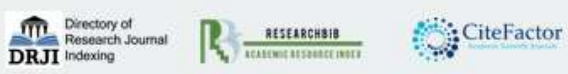

